\title{
Characteristics of Patients With Advanced Heart Failure Having Eosinophilic Infiltration of the Myocardium in the Recent Era
}

\author{
Investigation of Explanted Hearts for Transplantation \\ Saeko Yoshizawa, ${ }^{1 *} \mathrm{MD}$, Tomoko SugiYama Kato, ${ }^{2 *} \mathrm{MD}$, Donna Mancini, ${ }^{2} \mathrm{MD}$, \\ and Charles C. Marboe, ${ }^{1} \mathrm{MD}$
}

\begin{abstract}
SUMMARY
Eosinophilic infiltration of the myocardium is occasionally observed as an incidental histological finding in endomyocardial biopsy specimens before heart transplantation (HTx) as well as in explanted heart obtained at the time of HTx. However, the indications for HTx in these patients have not yet been fully established. We investigated the preHTx characteristics of the recipients with myocardial eosinophilic infiltration in the explanted heart and diagnosed as hypersensitivity myocarditis (HSM) (21 among 761 recipients, 2.8\%). Dobutamine, a common cause of HSM, was administered to 12 patients (57\%). Ten patients (47.6\%) were on milrinone and $4(19.0 \%)$ were on ventricular assist devices. Post-transplant survival of HSM patients was comparable to that of patients transplanted for active myocarditis or other cause of heart failure. In conclusion, myocardial eosinophilic infiltration is associated with multiple medications in patients with advanced heart failure; however, it does not affect the post-transplant prognosis. (Int Heart J 2013; 54: 146148)
\end{abstract}

Key words: Heart transplantation, Myocarditis, Eosinophils, Drugs

$\mathrm{T}$ he benefits of heart transplantation (HTx) for patients with myocarditis have been challenged because of possible recurrence and poor prognosis after HTx. Eosinophilic myocarditis, such as Chagas disease and ChurgStraus syndrome, was reported to have an influence on the prognosis after HTx, ${ }^{1,2)}$ whereas eosinophilic infiltration of the myocardium is occasionally observed as an incidental histologic finding in endomyocardial biopsy specimens before HTx as well as in explanted heart obtained at the time HTx. This is known as hypersensitivity myocarditis (HSM), which is a cardiac manifestation of a delayed-type allergic reaction typically caused by drugs. ${ }^{3,4)}$ The characteristics of patients with advanced heart failure and eosinophilic infiltration of the myocardium but a lack of any clinical manifestation of ChurgStraus syndrome or Chagas disease who were considered to have HSM, have yet to be elucidated in the recent era of heart failure medical therapy. Furthermore, the indication for HTx in patients coincidentally found to have HSM has not yet been fully discussed. Therefore, we investigated the incidence of HSM and its pre-transplant clinical characteristics using the database of a large transplant center.

\section{MeThods}

A total of 761 consecutive patients undergoing de novo HTx at Columbia University Medical Center were retrospectively reviewed. The pretransplant medication of HSM patients was examined. A diagnosis of HSM was made based on the histological evidence of the myocardium, that is mixed eosinophil-rich infiltrate with lymphocytes, macrophages, and plasma cells, but little myocyte necrosis as previously described. ${ }^{3)}$ The post-transplant survival rates of patients with HSM, those with other types of active myocarditis including giant cell myocarditis and lymphocytic myocarditis diagnosed by histological evaluation of the explanted heart $(n=31)$, and those with other causes of heart failure $(n=709)$ were compared using the Kaplan-Meier method with a log-rank test. All statistical analyses were performed using an SPSS software program (version 13.0J for Windows, SPSS Inc., Tokyo).

\section{RESUltS}

HSM was diagnosed in 21 patients (2.7\%) by histological evaluation of the explanted heart. None of them were suspect-

From the Departments of ${ }^{1}$ Pathology \& Cell Biology and ${ }^{2}$ Medicine, Division of Cardiology, Columbia University Medical Center and The New York Presbyterian Hospital, New York, USA.

* These authors contributed equally to this work.

Address for correspondence: Saeko Yoshizawa, MD, Department of Pathology \& Cell Biology, Columbia University Medical Center, 630 West 168th Street, PH 15W1574 New York, NY 10032, USA.

Received for publication February 14, 2013.

Revised and accepted March 4, 2013. 
ed of having HSM before HTx or diagnosed by endomyocardial biopsy performed as a part of an HTx candidacy evaluation. All patients with HSM had varying degrees of mixed inflammatory infiltrates with eosinophils, lymphocytes, macrophages, and plasma cells in the explanted hearts, but none of the patients exhibited myocardial necrosis (Figure 1). All except one patient with HSM were treated with inotropes before HTx; 12 patients $(57 \%)$ were on dobutamine and 10 (48\%) received milrinone (Table). When we performed a subanalysis of patients supported by dobutamine infusion but without LVAD support before HTx who were in United Network for Organ Sharing (UNOS) status 1A or 1B at the time of HTx $(n=372)$, patients with HSM were more likely to be treated with dobutamine before HTx compared to those with another cause of heart failure [HSM versus Active myocarditis versus Others = $12(57.1 \%)$ versus $6(37.5 \%)$ versus $53(14.9 \%), P=0.00001$; HSM versus Others, $P=0.00002$; HSM versus Active myocarditis, $P=0.236]$. Dobutamine was administered before HTx in 71 patients $(19.1 \%)$ in this cohort, and 12 patients $(16.9 \%)$ were diagnosed as HSM by histological examination of the explanted heart at the time of HTx. $\beta$-Blockers were administered to 7 patients $(33 \%)$ and ACE inhibitors to 11 patients (52\%). Antibiotics were administered to 12 patients, including 8 patients on vancomycin $(n=8,38.1 \%)$. Drugs that were reported to be associated with HSM such as heparin $(n=9)$, phenytoin $(n=1)$, and sulfonylurea $(n=1)$ were also used. ${ }^{5)}$ Four patients $(19.0 \%)$ were supported by left ventricular assist devices (LVAD) at the time of HTx. Seven (33\%) had a past history of drug allergy. Peripheral blood eosinophilia before HTx was observed in 19 patients (90\%). Peak eosinophil count averaged $900 / \mathrm{mm}^{3}$ with a range of 100 to 4000 (normal values less than $400 / \mathrm{mm}^{3}$ ). The post-transplant survival rates of patients with HSM, with active myocarditis, and with another cause of heart failure were not significantly different (Figure 2).

\section{DiscusSiON}

Eosinophilic infiltration to the myocardium, known as HSM, is a hypersensitivity response caused by a variety of drugs including inotropes, vasodilators, diuretics, and antibiotics. $^{3,4)}$ The incidence was reported to be $0.5 \%$ in autopsy series

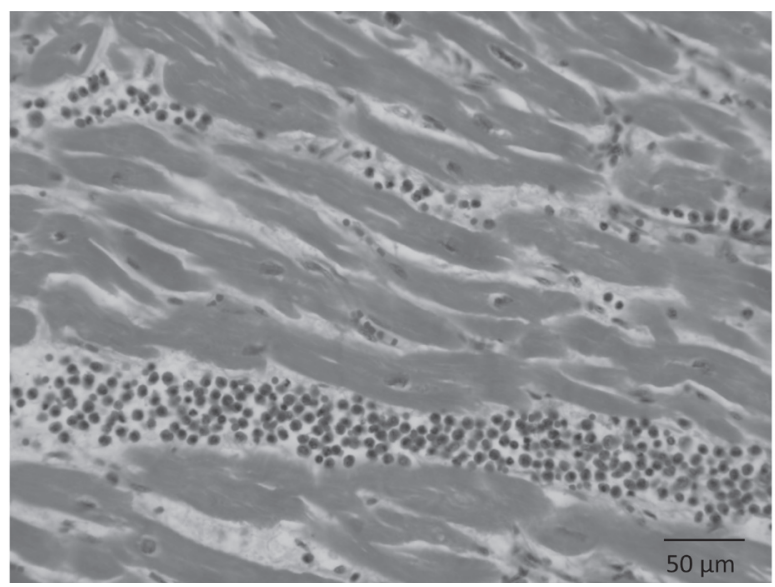

Figure l. Representative histological picture of hypersensitivity myocarditis (hematoxylin and eosin stain). and up to $23 \%$ in explanted heart for HTx. ${ }^{6,7)}$ Patients with advanced cardiac failure are generally treated with multiple medications, which is related to the increased incidence of HSM in HTx candidates. HSM may cause the symptoms of heart failure, ${ }^{8-10)}$ but most patients do not have obvious clinical features of HSM and they are frequently diagnosed as an incidental

Table. Pretransplant Medication of Patients With Hypersensitivity Myocarditis (HSM)

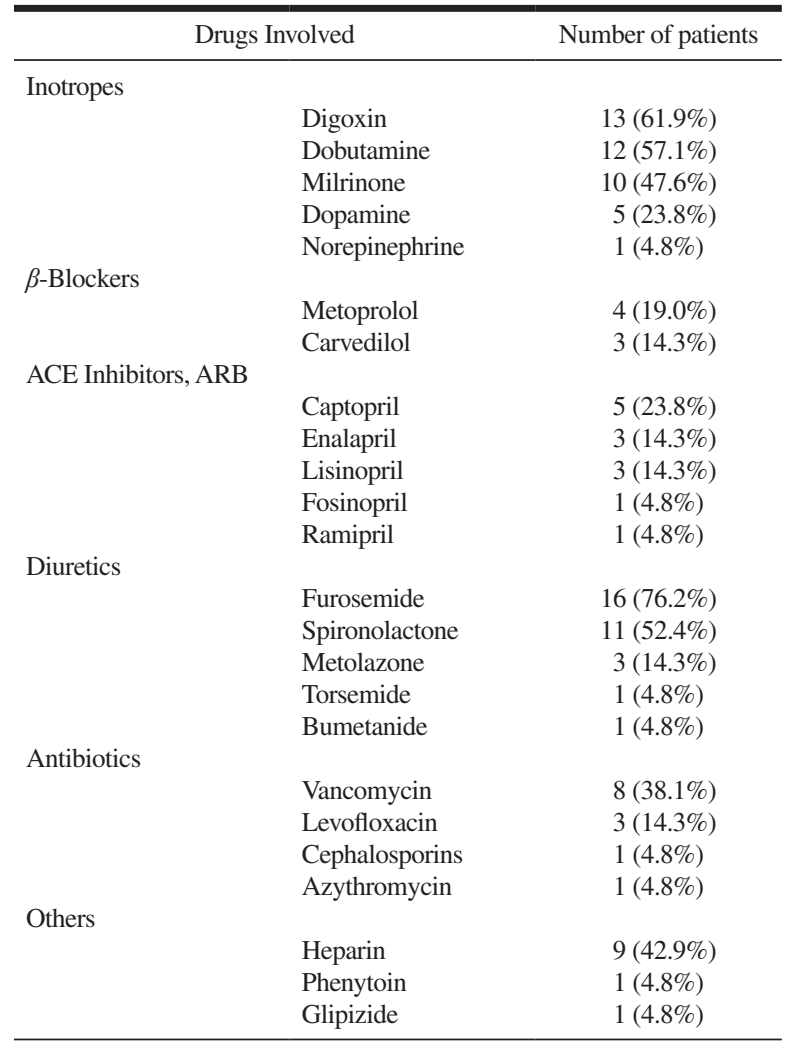

ACE indicates angiotensin converting enzyme and ARB, angiotensin II receptor blocker.

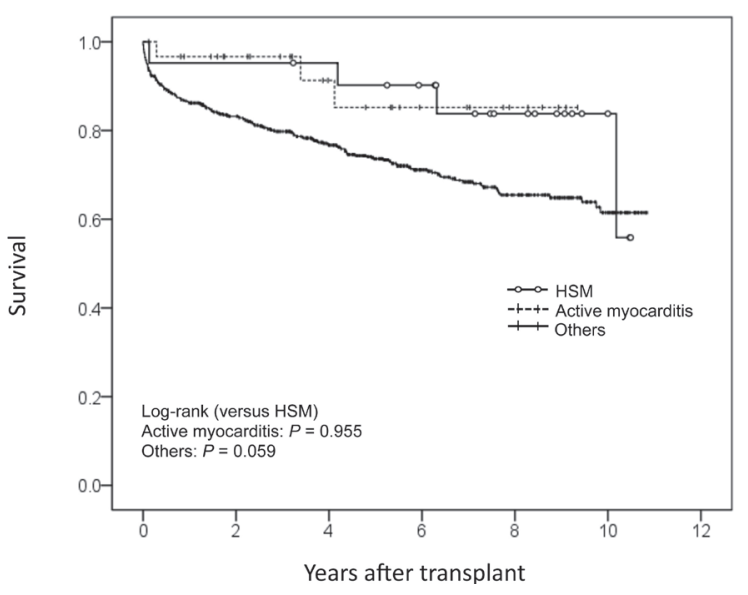

Figure 2. Kaplan-Meier survival curves of patients with hypersensitivity myocarditis (HSM) compared with that for the patients with active myocarditis and other diseases (Others). Groups did not differ with respect to all-cause mortality. 
finding at the time of HTx. HSM is usually resolved by drug withdrawal and corticosteroid therapy; however, it is difficult to wean heart failure patients from their medication even if HSM is diagnosed. Therefore, although the indication for HTx in patients with active myocarditis is still controversial, it may be difficult to exclude patients with HSM from the HTx waiting list. Little has been reported on the characteristics of patients with HSM in the era of modern medical therapy for heart failure, including LVAD usage and $\beta$-blocker administration as a standard medication.

In the present study, we found the incidence of HSM in a series of over 700 consecutive HTx recipients was about 3\%, which was lower than the incidence reported in previous studies. ${ }^{11,12)}$ We speculate that LVAD therapy has evolved into a standard therapy for patients waiting for HTx and it enables a majority of LVAD recipients to be weaned from continuous dobutamine infusion resulting in a low incidence of HSM among the total HTx recipient population, because dobutamine is a drug well-known to be associated with HSM. ${ }^{7,11)}$ Indeed, although the incidence of HSM itself was lower than in previous studies, nearly $60 \%$ of the patients with HSM in our cohort were on dobutamine. We also found that almost half of the patients received milrinone, specific phosphodiesterase type inhibitors, and one third of the patients were on $\beta$-blockers. Frustati, et al reported a case with HSM induced by metoprolol, ${ }^{13)}$ but the frequency of HSM caused by $\beta$-blockers has not yet been clarified. Because of multiple drug therapy, we could not determine which drug was involved in the development of HSM. However, we observed different trends in pharmacotherapy for heart failure from previous studies, which are worthwhile reporting in the era of recent heart failure therapy. We also observed the prognosis of patients with HSM was equivalent to those with myocarditis or other cause of heart failure as a primary reason for HTx.

The present study is a retrospective, single center analysis based on a small number of patients with HSM, and indeed, none of the patients were diagnosed as HSM during the transplant evaluation process. In addition, it may be difficult to distinguish HSM from myocarditis associated with hypereosinophilic syndrome, especially after treatment with steroids. However, we still believe that our investigation was conducted in a large cohort of heart-transplant recipients, since we are one of the largest transplant centers in the United States. Based on our study findings, we believe that patients with HSM would not necessarily need to be excluded from the waiting list for HTx because they can be expected to have a favorable life prognosis that is equal to other patients without HSM. Further studies would be required to evaluate the frequency of eosinophilic infiltration to the myocardium, which occurs during their heart failure treatment, and its effect on prognosis. In addition, the post-transplant prognoses of patients who are suspected to have eosinophilic infiltration to the myocardium should be investigated in large multi-institutional studies.

\section{REFERENCES}

1. Henderson RA, Hasleton P, Hamid BN. Recurrence of Churg Strauss vasculitis in a transplanted heart. Br Heart J 1993; 70: 553.

2. Campos SV, Strabelli TM, Amato Neto V, et al. Risk factors for Chagas' disease reactivation after heart transplantation. J Heart Lung Transplant 2008; 27: 597-602.

3. Fenoglio JJ Jr, McAllister HA Jr, Mullick FG. Drug related myocarditis. I. Hypersensitivity myocarditis. Hum Pathol 1981; 12: 900-7.

4. Burke AP, Saenger J, Mullick F, Virmani R. Hypersensitivity myocarditis. Arch Pathol Lab Med 1991; 115: 764-9.

5. Butany J, Ahn E, Luk A. Drug-related cardiac pathology. J Clin Pathol 2009; 62: 1074-84. (Review)

6. Johnson MR. Eosinophilic myocarditis in the explanted hearts of cardiac transplant recipients: Interesting pathologic finding or pathophysiologic entity of clinical significance? Crit Care Med 2004; 32: 888-90.

7. Spear GS. Eosinophilic explant carditis with eosinophilia: Hypersensitivity to dobutamine infusion. J Heart Lung Transplant 1995; 14: 755-60.

8. Zaacks SM, Klein L, Tan CD, Rodriguez ER, Leikin JB. Hypersensitivity myocarditis associated with ephedra use. J Toxicol Clin Toxicol 1999; 37: 485-9.

9. Chikwava KR, Savell VH Jr, Boyd TK. Fatal cephalosporin-induced acute hypersensitivity myocarditis. Pediatr Cardiol 2006; 27: 777-80.

10. Frustaci A, Verardo R, Sale P, et al. Hypersensitivity myocarditis induced by beta-blockers: an unexpected cause of abrupt deterioration in hypertrophic cardiomyopathy. Intensive Care Med 2007; 33: 1848-9.

11. Takkenberg JJ, Czer LS, Fishbein MC, et al. Eosinophilic myocarditis in patients awaiting heart transplantation. Crit Care Med 2004; 32: 714-21.

12. Hawkins ET, Levine TB, Goss SJ, Moosvi A, Levine AB. Hypersensitivity myocarditis in the explanted hearts of transplant recipients. Reappraisal of pathologic criteria and their clinical implications. Pathol Annu 1995; 30: 287-304. (Review)

13. Frustaci A, Verardo R, Sale P, et al. Hypersensitivity myocarditis induced by beta-blockers: an unexpected cause of abrupt deterioration in hypertrophic cardiomyopathy. Intensive Care Med 2007; 33: $1848-9$. 